\title{
TEXTURES IN CERAMIC SUPERCONDUCTORS
}

\author{
W. P. LIU, N.-J. PARK and H. J. BUNGE \\ Department of Physical Metallurgy, Technical University Clausthal, Germany
}

(Received 30 April 1990)

Textures in bulk $\mathrm{Y}-\mathrm{Ba}-\mathrm{Cu}-\mathrm{O}$ superconducting materials were studied. Texturing methods are based on the paramagnetic anisotropy, grain-dimension anisotropy and surface-energy anisotropy of the crystals. Five incomplete $\mathrm{X}$-ray pole figures including four superimposed ones were measured for the ODF calculation. The ODFs were calculated with the series expansion method including the positivity method by means of an iteration process.

The results show that a strong (001)-fibre texture can be achieved by magnetic alignment but the density of the materials after sintering is still too low. The grain-dimension effect which exists in nearly all powder-forming process, is very weak for texturing of the materials. The surface texture depends on recrystallization of the powder particles.

KEY WORDS Y-Ba-Cu-O, powder sintered bulk material, magnetic alignment, surface texture, superposed pole figures, ODF-calculation.

\section{INTRODUCTION}

Since single crystals of high temperature superconductors exhibit a large anisotropy, the orientation distribution of crystallites in polycrystalline materials is an important factor to determine the superconducting properties, such as critical current which is one of the main limitations that stand in the way to practical applications.

Textures in powder-sintered bulk materials can be achieved by different methods. These methods are mainly based on three principles:

Magnetic anisotropy. As is well known, powder particles of ceramic superconductors tend to align with a crystal $c$-axis parallel to the magnetic field because of paramagnetic anisotropy (Farrell et al., 1987). If a mixture of the ceramic powder and a suitable binder is put into a magnetic field, a strong texture in the composite can be obtained. After sintering, the binder is burnt away and the particles remain in the preferred orientation.

Grain dimension anisotropy. Another principle of texturing is based on the particle aspect-ratio effect. Because powder particles of ceramic superconducting materials often show a plate-like form, thin in $c$-axis direction, they can be aligned by a mechanical movement during powder forming with their $c$-axis parallel to, e.g. pressing direction. Some techniques of texturing, such as hot pressing and hot extrusion (Chen et al., 1987), sinter forging (Robertson et al., 1987), cold extrusion (Rajan et al., 1989) and uniaxial cold pressing (Liu et al., 1989) might be based on this effect.

Surface-energy anisotropy. It has been found (Liu et al., 1989) that a 
(001)- fibre texture usually exists on the sample surface after sintering. This may be attributed to low surface energy of the (001) plane. The surface texture is limited to about one grain diameter below the surface.

In order to compare the texturing principles mentioned above, the textures developed by different methods were determined quantitatively. Since the properties of ceramic superconducting materials are similar with respect to the above mentioned three principles, only $\mathrm{Y}-\mathrm{Ba}-\mathrm{Cu}-\mathrm{O}$ material was used. The samples were prepared by magnetic alignment and free rolling which is a method based on the aspect-ratio effect. The surface texture of uniaxially pressed samples was also measured. The orientation distribution functions (ODF) were calculated from measured pole figures using the series expansion method (Bunge, 1982) including positivity method (Dahms, 1989).

\section{EXPERIMENTAL PROCEDURE}

The magnetically aligned samples were prepared of 3-5 $\mu \mathrm{m}$ powder of $\mathrm{Y}-\mathrm{Ba}-$ $\mathrm{Cu}-\mathrm{O}$ material and epoxy resin. They were held in a $1.8 \mathrm{~T}$ magnetic field for a few hours and then sintered at $950^{\circ} \mathrm{C}$.

The so-called free-rolling method was chosen to test the aspect-ratio effect. The samples were prepared by mixing $\mathrm{Y}-\mathrm{Ba}-\mathrm{Cu}-\mathrm{O}$ powder and $10 \mathrm{wt} \%$ epoxy resin. The mixture was then wrapped with $0.5 \mathrm{~mm}$ thick lead foil and rolled with small reductions in thickness per pass down to $70 \%$ reduction. Because of the soft $\mathrm{Pb}$-foil and the organic binder as lubricant, the powder particles can move freely during the rolling process. They rotate into the preferred orientation, that is, with their $c$-axes perpendicular to the rolling plane. After rolling the $\mathrm{Pb}$-foil was removed. The organic binder was burnt away during subsequent sintering for $12 \mathrm{hrs}$ at $950^{\circ} \mathrm{C}$ in air.

The surface texture was measured at the sample surface after uniaxial pressing and sintering for $12 \mathrm{hrs}$ at $950^{\circ} \mathrm{C}$ in air.

Five incomplete pole figures, i.e., (110) $+(103)+(013),(005)+(104)+(014)$, (113), $(006)+(200)+(020)$ and $(116)+(123)+(213)$ were measured in steps $\Delta \alpha=5^{\circ}$ and $\Delta \beta=3.6^{\circ}$ up to $\alpha=70^{\circ}$ using an automatic X-ray texture goniometer, ATEMA-C.

\section{ODF CALCULATION}

The studied material, $\mathrm{Y}-\mathrm{Ba}-\mathrm{Cu}-\mathrm{O}$, has orthorhombic crystal symmetry, the lattice parameters being

$$
a=3.82 \AA, b=3.88 \AA, c=11.68 \AA
$$

Hence, the reflexions $(h k l)$ and $(k h l)$ are systematically superimposed. Furthermore, the crystallites are heavily twinned with (110) as the twinning plane. Hence, orientations rotated $90^{\circ}$ about the $c$-axis are nearly equally frequent in the texture. Because of these two reasons it seemed justified to treat the material as being pseudo-tetragonal. Even with this symmetry there are not enough non-overlapped pole figures available to calculate the ODF. Hence, it was 
essential to use an ODF calculation procedure which allows the use of superimposed pole figures.

For superimposed pole figures one has to know the intensity-coincidence factors. A concident pole figure measured at a Bragg angle $2 \theta$ can be represented by a linear combination of several pole figures:

with

$$
P_{\theta}(\alpha \beta)=\sum_{i=1}^{I} q_{i} P_{h k l_{i}}(\alpha \beta)
$$

$$
\sum_{i=1}^{I} q_{i}=1
$$

where $q_{i}$ are the intensity-coincidence factors, if the crystal structure is well known and the reflections are totally coincident, the $q_{i}$ can be calculated from the squares of the structure factors and the multiplicity factors of the reflections. Since, however, in $\mathrm{Y}-\mathrm{Ba}-\mathrm{Cu}-\mathrm{O}$ materials after rapid cooling the position of oxygen atoms is uncertain, the structure factors can not be calculated precisely and hence the coincidence factors are unknown. In our case, the pole figures were measured up to $\alpha=70^{\circ}$. For such incomplete and coincident pole figures the normalization factors, $N$, are also unknown.

In order to solve this problem, a first approximation of the normalization factors is obtained by extrapolating the measured pole figures up to $\alpha=90^{\circ}$ and taking a suitable approximation for the coincidence factors, $q_{i}$, then the approximate texture coefficients, $C_{l}^{\mu \nu}$, can be determined by means of a least squares method: (Dahms, 1989)

$$
\begin{aligned}
\sum_{j=1}^{J} \int_{\alpha=0}^{\alpha \max } \int_{\beta=0}^{2 \pi}\left[P_{\theta_{j}}(\alpha \beta)-\sum_{i=1}^{I} q_{i_{j}} \sum_{l=0}^{L} \sum_{\mu=1}^{M(l)} \sum_{\nu=0}^{N(l)}\right. \\
\left.\frac{4 \pi}{2 l+1} C_{l}^{\mu \nu} \dot{k}_{l}^{\mu}\left(h k l_{i j}\right) \dot{k}_{l}^{v}(\alpha \beta)\right]^{2} \sin \alpha \mathrm{d} \alpha \mathrm{d} \beta=\min
\end{aligned}
$$

that is, the deviation of the assumed texture with respect to all measured pole figures including coincident ones is minimized. From the approximate coefficients $C_{l}^{\mu \nu}$, the pole figures, $P_{h k l}(\alpha \beta)$, can be re-calculated. With the help of positivity condition (the pole density in pole figures must not be negative, i.e. $P_{h k l}(\alpha \beta) \geqslant$ 0 .) the re-calculated pole figures can be further corrected. They are used in the non-measured range of the pole figures to obtain the next approximation of $N$ and $q$. This procedure can be repeated as many times as necessary until no further improvement can be reached. Figure 1 is the block diagram of the calculation process.

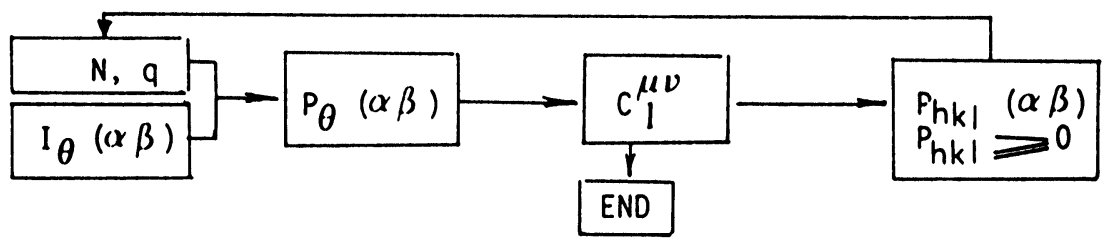

Figure 1 Block diagram of the iteration process for ODF calculation. 


\section{RESULTS AND DISCUSSION}

As an example we have calculated the ODF of a magnetically aligned $\mathrm{Y}-\mathrm{Ba}-\mathrm{Cu}-$ $\mathrm{O}$ sample before sintering. Five pole figures including 4 coincident ones were used as input. The iteration process was repeated up to 60 times according to Figure 1. The ODF of this state is shown in Figure 2.

Figure 3 shows the experimentally measured and the re-calculated pole figures. Comparing these pole figures, it can be seen that they are very similar and their maximum density values correspond to each other quite well, e.g., the maximum value of the (113) pole figure is 2.49 for the experimental and 2.31 for the re-calculated one. In addition, the coincident pole figures are well separated.

As has been shown in Figure 2, the "green" sample before sintering has a strong fibre texture with the (001)-axis parallel to the magnetic field. The maximum value of $f(g)$ is $30.78 \times$ random. After sintering, the texture becomes weaker, as is shown in Figure 4. The maximum value of $f(g)$ decreases to $22 \times$ random. In addition, the texture deviates a little from pure fibre texture.

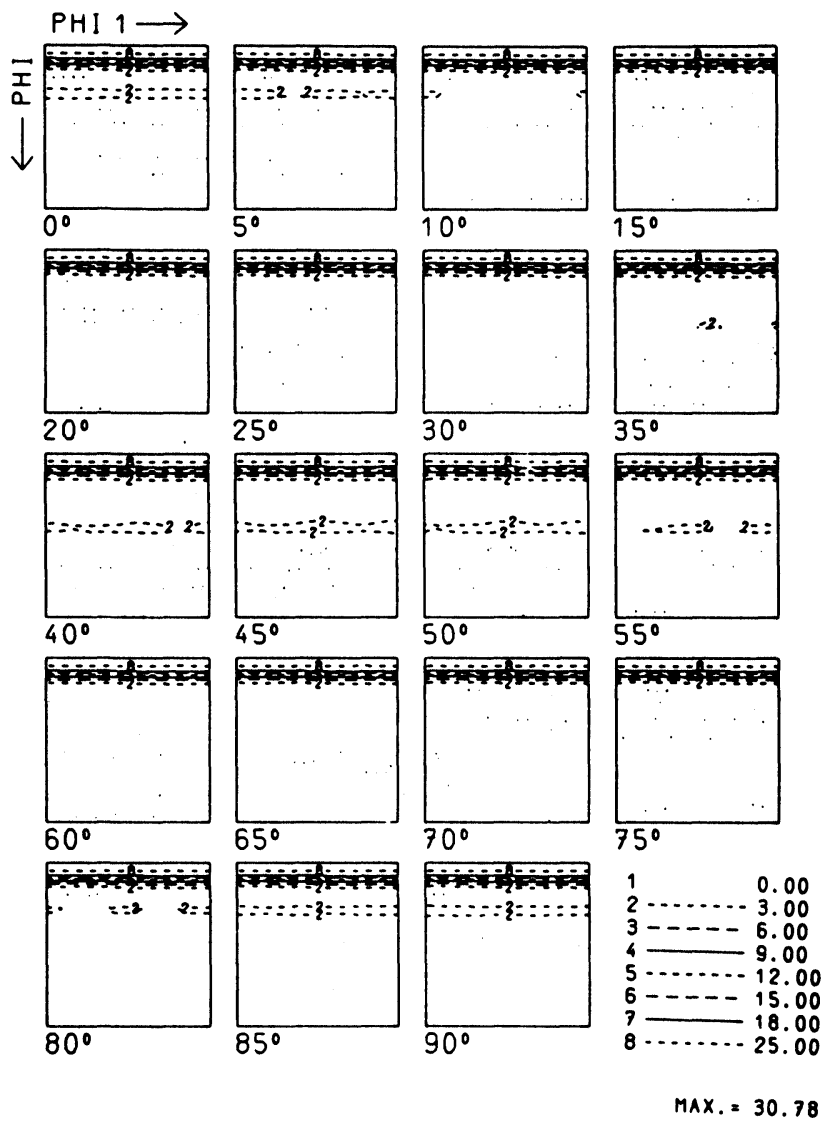

Figure 2 ODF of a magnetically aligned $\mathrm{Y}-\mathrm{Ba}-\mathrm{Cu}-\mathrm{O}$ sample before sintering $\left(L_{\max }=18\right)$. 
(a)

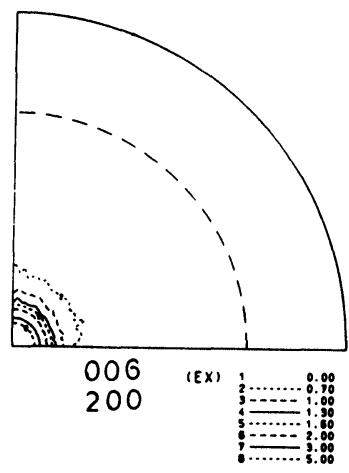

(b)

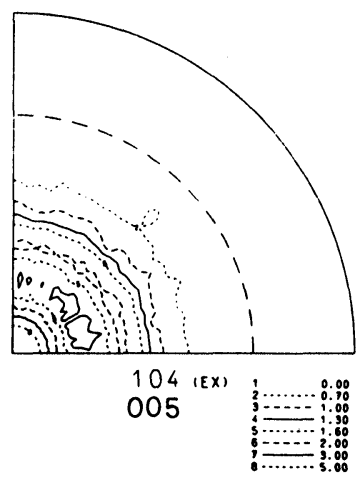

(c)

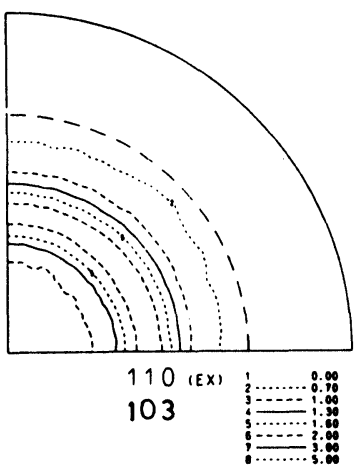

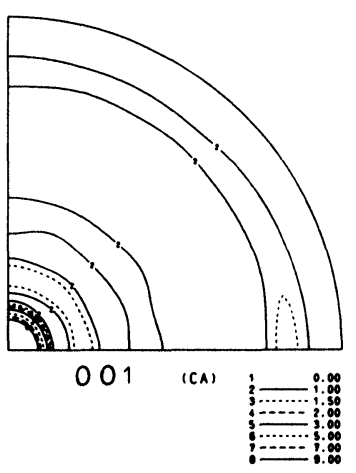

$\max .090 .02$
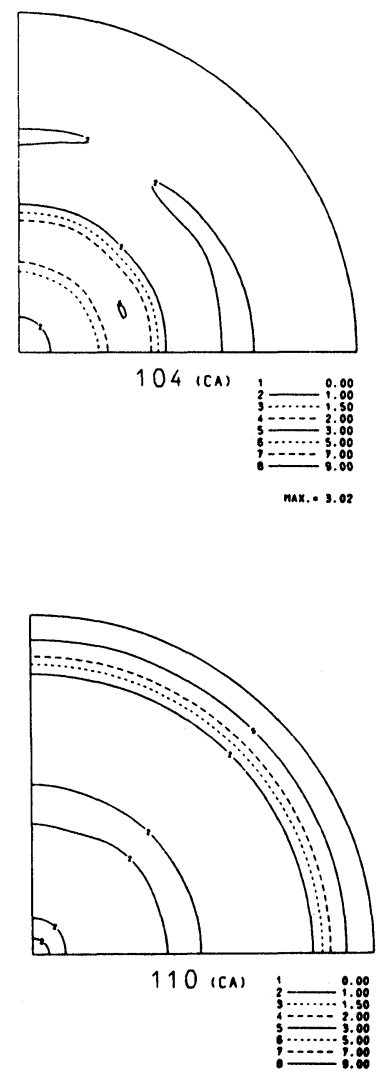

nax. - 3.
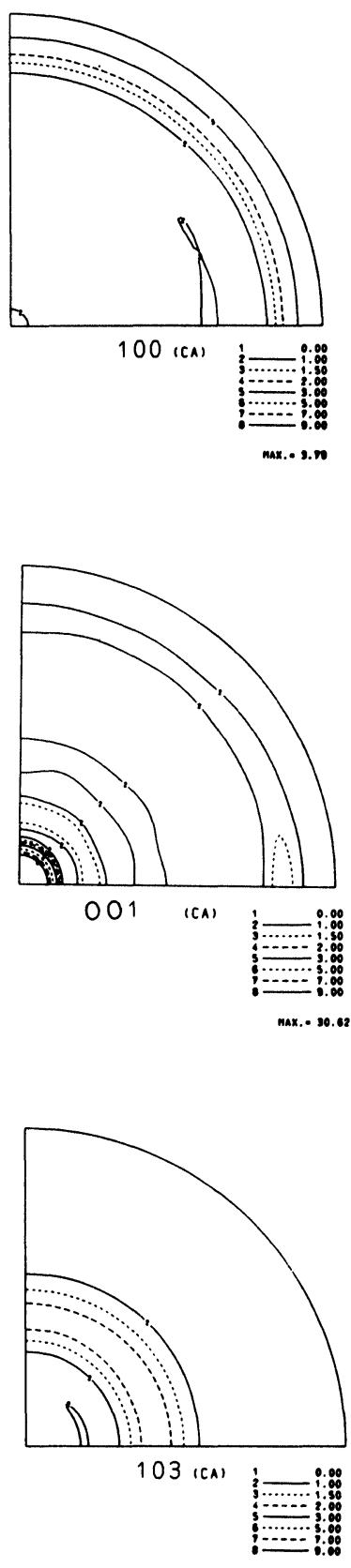

$\max , \cdot 2.33$

Figure 3 (a) Experimentally measured (006) + (200) coincident pole figure (left) and re-calculated, separated (001) and (100) pole figures. (b) Experimentally measured (005) $+(104)$ coincident pole figure (left) and re-calculated, separated (104) and (001) pole figures. (c) Experimentally measured $(110)+(103)$ coincident pole figure (left) and re-calculated, separated (110) and (103) pole figures. 
(d)

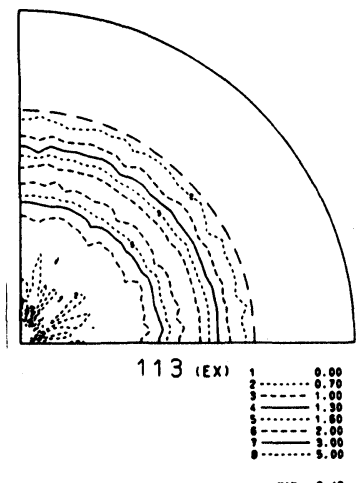

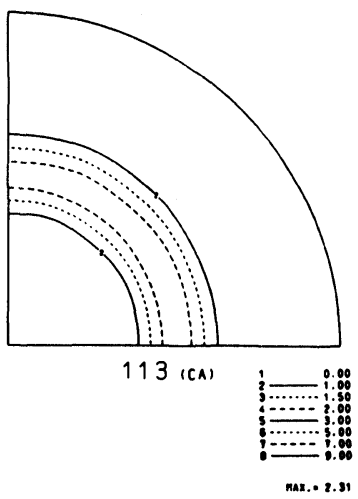

Figure 3 (Continued) (d) Experimentally measured (left) and re-calculated (113) pole figures.

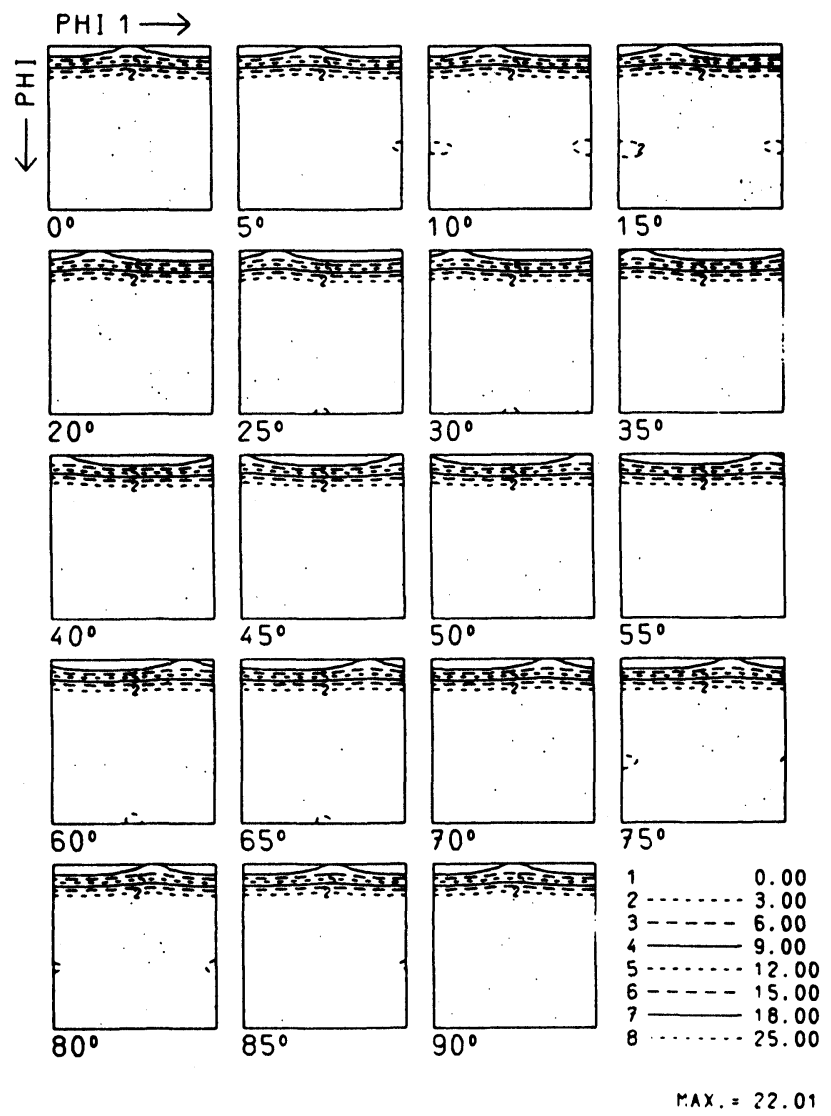

Figure 4 ODF of the magnetically aligned $\mathrm{Y}-\mathrm{Ba}-\mathrm{Cu}-\mathrm{O}$ sample after sintering. $\left(L_{\max }=14\right)$. 


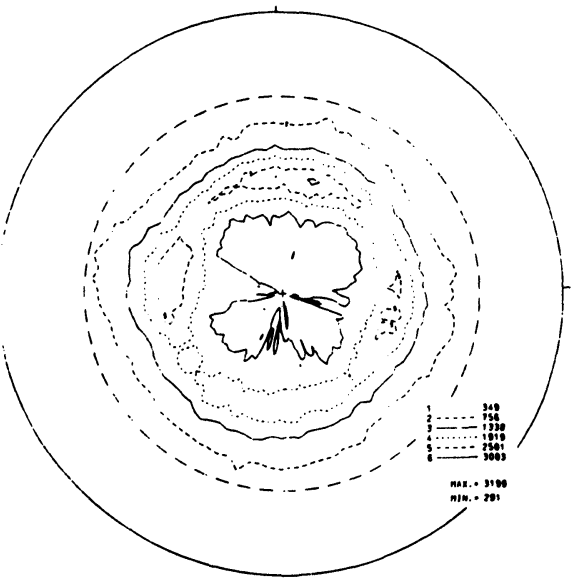

(a)

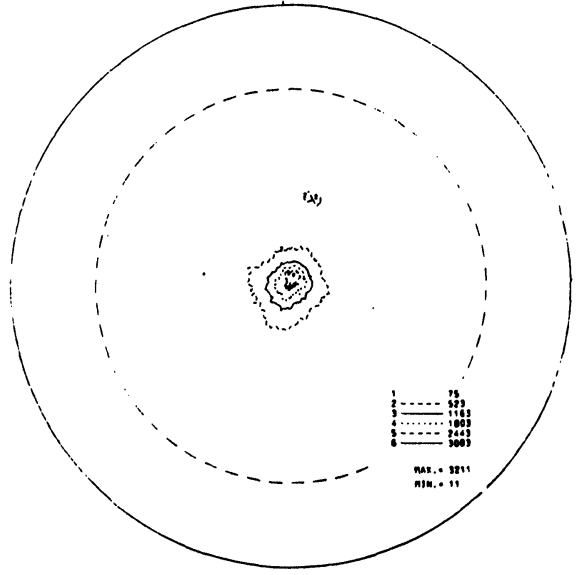

(b)

Figure 5 Pole figures of the magnetically aligned sample after sintering. (a) (103) $+(110)$. (b) (005) + (104)

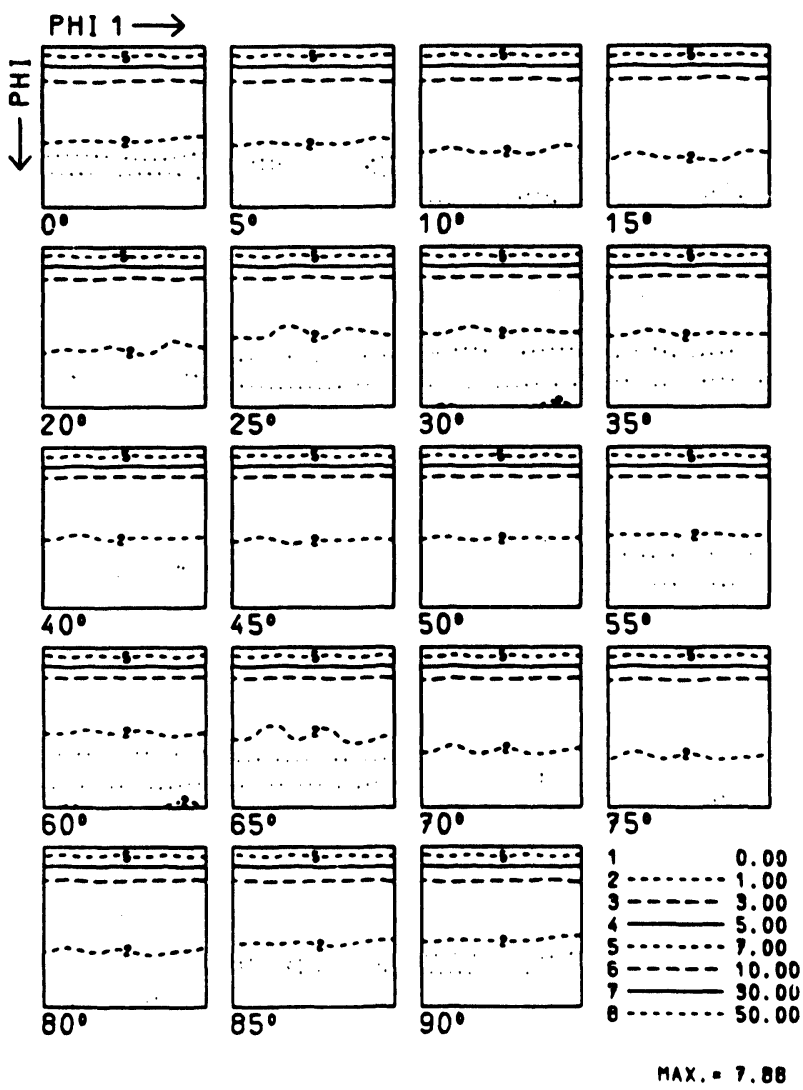

Figure 6 ODF of the surface texture $\left(L_{\max }=14\right)$. 


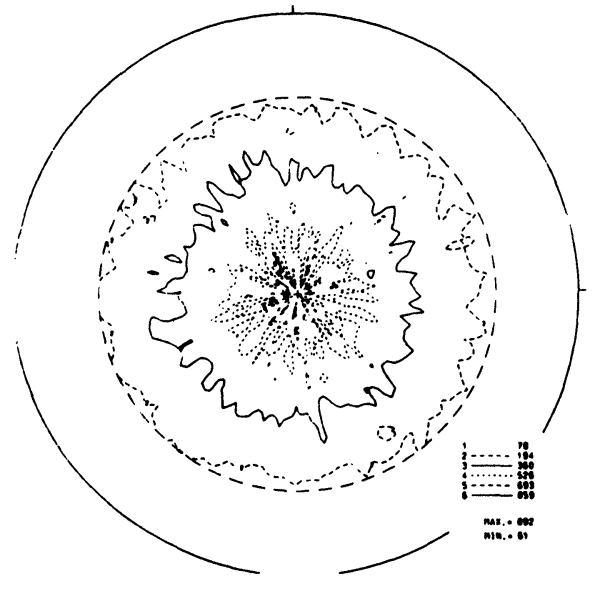

(a)

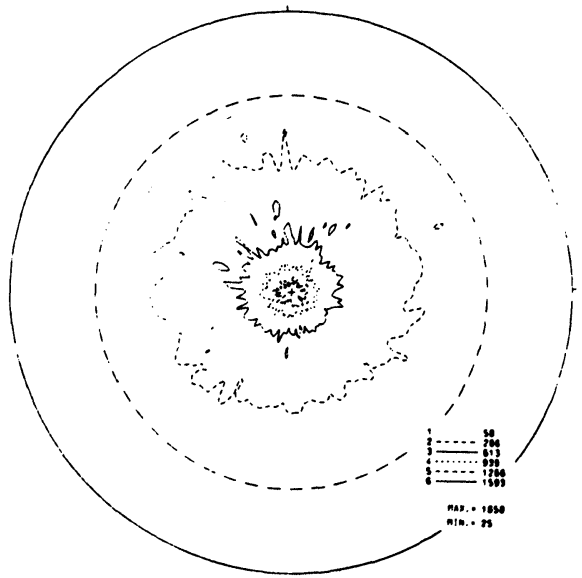

(b)

Figure $7(005)+(104)$ pole figures of the surface texture. (a) after removing the surface layer and re-annealing (b) original surface texture.

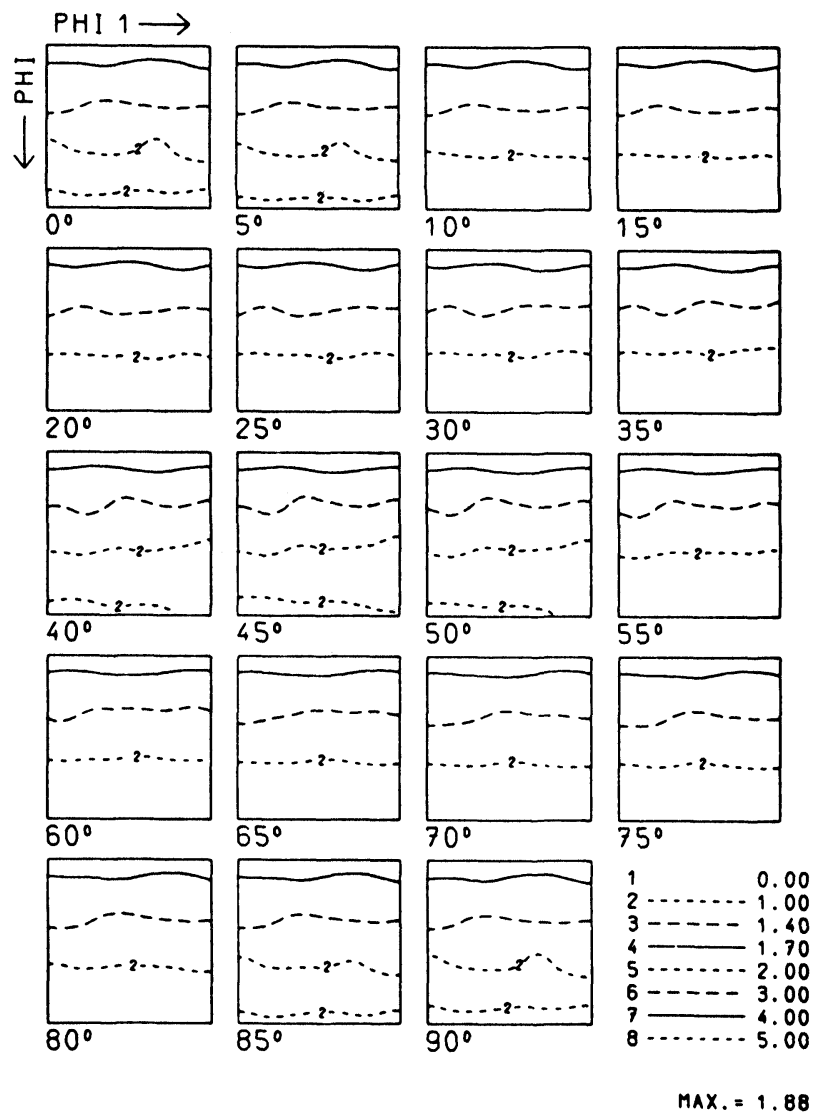

Figure 8 ODF of a free-rolling sample $\left(L_{\max }=12\right)$ 
Since the intensity distribution in pole figures (Figure 5) is similar to the aspect of the sample (in our case the sample is a square plate), the deviation from pure fibre texture after sintering may be caused by anisotropic shrinkage of the sample during sintering. After sintering the density of the material was $3-3.5 \mathrm{~g} / \mathrm{cm}^{3}$, i.e. only a half of the theoretical density. This is too low for practical application. Ways may be found, however, to increase the density and at the same time the texture, too.

Figure 6 shows the ODF of the surface texture of a pressed sample. It is also a (001)-fibre texture. The maximum value of $f(g)$ is $7.88 \times$ random. Surface texture was only found after sintering of the pressed powder sample. If the surface layer (with surface texture) was removed and the sample was annealed at $950^{\circ} \mathrm{C}$ for $12 \mathrm{hrs}$ once again, the strong surface texture appeared no more. Figure 7a shows the $(005)+(104)$ pole figure measured from the surface of a re-annealed sample. Comparing with the surface texture of the powder-sintered sample (Figure 7b) it is clear that the strong surface texture develops only by the first powder sintering. Hence, it may be concluded that the defect structure of the powder particles after grinding provides a driving force for grain growth which is no more present in the second annealing process.

The (001)-fibre texture of a free-rolled sample (Figure 8 ) is very weak. The maximum value of $f(g)$ is $1.88 \times$ random. It is only slightly stronger than that of a uniaxially pressed sample, as is seen in the pole figures shown in Figure 9. Figure 10 finally shows the $(006)+(200)$ pole figures of the free-rolled sample, the surface texture and the magnetically aligned sample. It can be seen that the aspect-ratio effect, which is the fundamental of many texturing methods accomplished during powder-forming process, is too weak to achieve a sharp enough texture. But, nevertheless, texture does exist in free-rolled (and uniaxially pressed) samples and it is strong enough to affect the intensities of $2 \theta$-diffraction scans and, perhaps, to affect the properties of the materials to some extent.

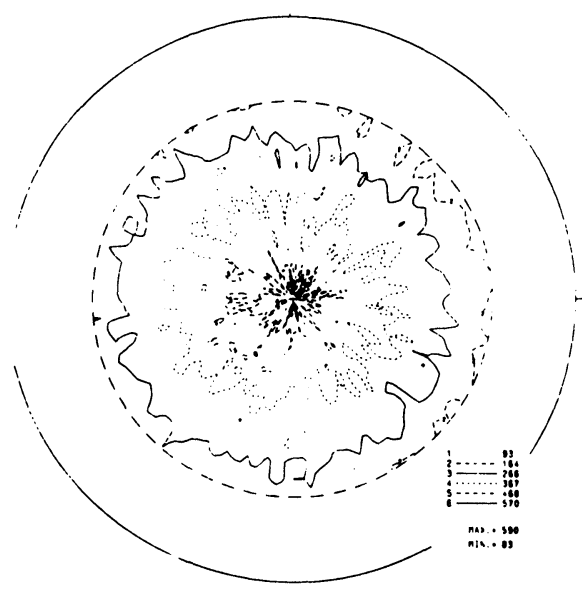

(a)

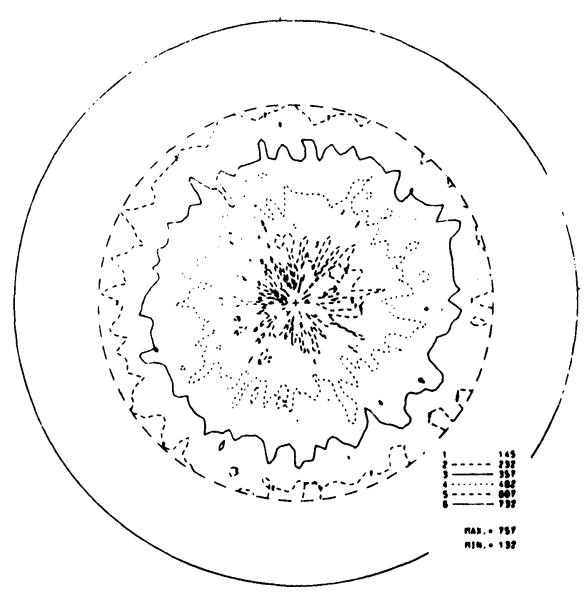

(b)

Figure $9(005)+(104)$ pole figures of a free-rolling sample (a) and a uniaxially pressed sample (b). 
(a)

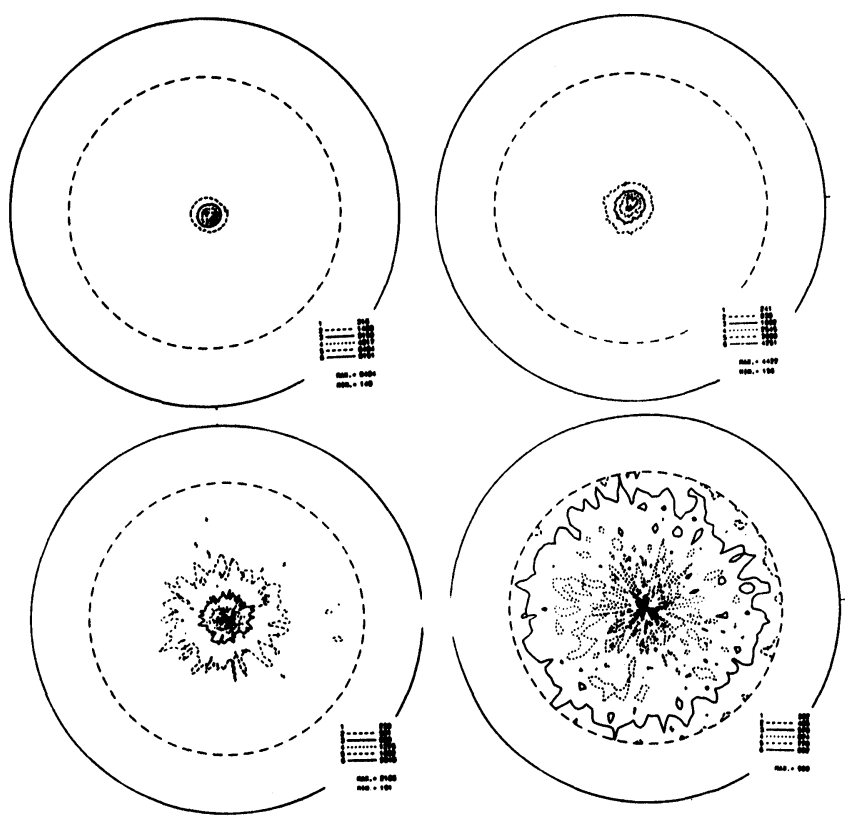

(b)

(c)

Figure $10(006)+(200)$ pole figures of the magnetically aligned sample before sintering (a), magnetically aligned sample after sintering (b), surface texture (c) and free-rolling sample (d).

\section{References}

Bunge, H. J. (1982) Texture Analysis in Materials Science, Butterworth, London.

Chen, I.-W., Wu, X., Keating, S. J., Keating, C. Y., Johnson, P. A. and Tien, T.-Y. (1987) Texture development in YBaCuO by hot-extrusion and hot-pressing. J. Am. Ceram. Soc. 70(12), 388.

Dahms, M. and Bunge, H. J. (1989) The iterative series-expansion method for quantitative texture analysis. I general outline, J. Appl. Cryst. 22, 439-447.

Farrell, D. E., Chandrasekhar, B. S., DeGuire, M. R., Fang, M. M., Kogan, V. G., Clem, J. R. and Finnemore, D. K. (1987) Superconducting properties of aligned crystalline grains of $\mathrm{YBaCuO}$, Phys. Rev. B36, 4025.

Liu, W. P., Park, N.-J. Bunge, H. J. (1989) Textures in powder-sintered YBaCuO superconductors. In: Modern Aspects of Superconductivity, ed. R. Suryanarayanan, IITT International, France, 107.

Rajan, K., German, R. M., Knorr, D. B., MacCrone, R. K., Misiolek, W. and Wright R. N. (1989) Deformation processing of high-Tc superconducting oxides, JOM. April 1989, 28.

Robertson, Q., Georgopoulos, P., Johnson, D. L., Marcy, H. O., Kannewurf, C. R., Hwu, S. J., Marks, T. J., Poeppelmeier, K. R., Song, S. N. and Ketterson, J. B. (1987) Sinter-Forged YBaCuO, Adv. Ceram. Mater. 2(3B), 380. 